\title{
LAS ELECCIONES PRESIDENCIALES \\ DE 1995 EN FRANCIA
}

JUAN MANUEL GOIG MARTÍNEZ

Profesor Asociado del Departamento de Derecho Constitucional

UNED 


\section{SUMARIO}

I. EL PRESIDENTE DE LA REPÚBLICA FRANCESA.-1. Evolución del sistema de elección presidencial.-2. El sistema de elección presidencial.-3. Los poderes del presidente de la república.-II. LA CAMPAÑA PRESIDENCIAL DE 1995.-1. Los candidatos presidenciales en campaña. - 2. Sondeos electorales.-3. Resultados electorales en la primera vuelta.-III. LA SEGUNDA VUELTA EN LAS ELECCIONES PRESIDENCIALES DE 1995.-1. Las consecuencias del "ballotage».-2. Resultados electorales. 


\title{
LAS ELECCIONES PRESIDENCIALES DE 1995 EN FRANCIA
}

\author{
POR \\ JUAN MANUEL GOIG MARTINNEZ \\ Profesor Asociado del Departamento \\ de Derecho Constitucional \\ UNED \\ I. EL PRESIDENTE DE LA REPÚBLICA FRANCESA
}

\section{Evolución del Sistema de Elección Presidencial}

Mucho ha evolucionado el sistema de elección presidencial en la República francesa desde la Constitución de 4 de noviembre de 1848, cuyos artículos 45 y 46 establecían que el Presidente de la República sería elegido por 4 años, mediante escrutinio secreto y por mayoría absoluta "por todos los electores de los departamentos franceses y de Argelia", pudiendo ser reelegido tras un receso de 4 años.

Las Leyes Constitucionales de 1875 de la III República francesa concedía a la Cámara de Diputados y al Senado, reunidos en Asamblea Nacional, la potestad de elegir al Presidente, por 7 años, por mayoría absoluta '. La Constitución de la IV República, Constitución de 27 de octubre de 1946, ponía en manos de la Asamblea Nacional y del Consejo de la República, reunidos en Parlamento, la función de elección presidencial (artículo 29).

1 Artículo 2 de la Ley de 25 de febrero de 1875. 
Los constituyentes de 1958, confieren la elección presidencial a un colegio, compuesto por ciudadanos que detentan un mandato electivo, influídos por las ideas expuestas por de Gaulle, quien el 21 de diciembre de 1958 sería designado Presidente de la República francesa mediante este procedimiento. No obstante, el mismo de Gaulle, propuso, en el año 1962, la reforma de los artículos 6 y 7 de la Constitución, a fin de proceder a dar efectividad al principio de elección presidencial mediante sufragio universal, escogiendo para esta reforma la vía del referéndum prevista en el artículo 11, referendum que sería celebrado, y aprobada la propuesta, el 28 de octubre de 1962, en lugar del procedimiento de reforma constitucional fijado en el artículo $89^{2}$.

La ley de 6 de noviembre de 1962 modifica los artículos 6 y 7 de la Constitución incorporando el sufragio universal como forma de elección presidencial.

Tras las elecciones de 1974, provocadas por la muerte de Georges Popidou, ante el vacio constitucional referente a la posibilidad de muerte de un candidato durante el período electoral, el Consejo Constitucional solicita respuesta a los problemas derivados de esta elección, en virtud de la cual, el artículo 7 de la Constitución fue, de nuevo, modificado ${ }^{3}$.

\section{El Sistema de Elección Presidencial}

Según la Constitución francesa, podrá presentarse a la Presidencia de la República cualquier ciudadano que, estando en posesión de sus plenos derechos civiles, sea mayor de 23 años, sin que sea preciso el apoyo de ningún partido político, aunque lo más común consiste en la postulación de dirigentes políticos.

Las candidaturas deberán comunicarse al Consejo Constitucional, órgano de control electoral, antes de la medianoche del décimo noveno día anterior al primer escrutinio, procediéndose al depósito de una cantidad dineraria que será reembolsada si se obtuviera, al menos, el 5 por 100 de los

2 La opción por la vía de referendum provocaría que el Presidente del Senado considerara la reforma como una violación del texto constitucional y planteara la inconstitucionalidad ante el Consejo Constitucional francés, quien, mediante decisión no 62-20 DC, Rec. p. 28, se inhibió en el asunto.

1976.

3 Ley Constitucional no 76-527 de 18 de junio de 1976, JO de 19 de junio de 
votos emitidos. Con el fin de asegurar una cierta seriedad, cada candidato deberá contar con el apoyo de, al menos, 500 personas que desempeñen puestos de elección ${ }^{4}$, para lo cual, el Gobierno francés envía 15 días antes de la publicación del aviso que constituye el anuncio oficial de la elección, a cada persona que desempeña un cargo público, el formulario pertinente.

A fin de facilitar la igualdad de oportunidades a todos los candidatos, el Estado les asigna la cantidad de 1.000 francos, como gastos de campaña, así como se hace cargo del costo de carteles y circulares, cuyo número y tamaño están regulados por ley, siendo obligatorio, desde 1988 que los partidos políticos den a conocer los gastos ocasionados por la campaña electoral.

Según el artículo 7 de la Constitución, "el Presidente de la República es elegido por la mayoría de los votos emitidos. Si esta mayoría no es obtenida en el primer turno de votos, se procederá a una segunda vuelta el segundo domingo siguiente». No obstante, a la segunda vuelta sólo podrán optar los dos candidatos que hayan resultado más favorecidos por el recuento de los votos de la primera vuelta.

El Presidente es elegido por un período de 7 años, pero puede ser reelegido para otro período subsiguiente, habiéndose eliminado la imposibilidad de reelección se reduce una situación peculiar en un régimen político de carácter semipresidencialista ${ }^{6}$.

En lo que a la duración del mandato se refiere, la postura de los principales candidatos presidenciales dista mucho, puesto que mientras Chirac defiende el mantenimiento de los 7 años de mandato, al considerar que su reducción la haría coincidir con el mandato parlamentario, lo que situaría el sistema político francés en el más puro estilo presidencialista, el candidato socialista Jospin propugnaba como punto importante de su campaña, en lo que a las instituciones se refiere, la reforma constitucional que redujera a 5 años el mandato presidencial por considerar el septenato un periodo demasiado largo en el quehacer del Presidente.

4 Una de las características más interesantes de la reforma electoral aprobada el 6 de noviembre de 1962, consistia en la necesidad de que los candidatos presidenciales fueran recomendados por un número no inferior a 100 cargos electos, cantidad que fue modificada por la Asamblea Nacional, mediante la Ley Orgánica $\mathrm{n}^{\circ} \mathbf{7 6 - 5 2 8}$ de 18 de junio de 1976, y elevada a 500, entrando este sistema en vigor en 1981.

5 Mediante Ley Orgánica no 88-226 de 11 de marzo de 1988 relativa a la trasparencia financiera de la vida política, JO de 12 de marzo de 1988.

- NúNEEz RIVERo C.: "El Régimen político francés» en Introducción al Derecho Político UNED, Madrid, 1993, pág. 522. 


\section{Los poderes del Presidente de la República}

El Presidente de la República vela por el respeto de la Constitución. Asegura, a través de su arbitrage, el funcionamiento regular de los poderes públicos, así como la continuidad del Estado (artículo 5).

La noción de árbitro contenida en el texto constitucional debe ser entendida en un sentido extensivo, puesto que el Presidente no ejerce una magistratura de influencia, hecho que viene demostrado por las importantes prerrogativas que le son otorgadas sin la exigencia de refrendo ministerial (artículo 19.

\section{Poderes propios:}

Corresponde al Presidente de la República:

- El nombramiento del Primer Ministro. Para el ejercicio de esta función cuenta el Presidente con una total libertad, cuando menos teórica, ya que no necesita abrir consultas con los representantes de los grupos parlamentarios. No obstante, la posibilidad de que la Asamblea Nacional pueda obligar a dimitir a los Primeros Ministros de designación presidencial, determina que el Presidente deba tener en cuenta la proporcionalidad de las fuerzas parlamentarias. Las relaciones Presidente-Primer Ministro, se determinarán por la existencia o no de "cohabitación política".

- Convocar a referendum.

- Disolver la Asamblea Nacional.

- Plantear la constitucionalidad ante el Consejo Constitucional.

- Nombrar a tres miembros del Consejo Constitucional.

- Elevar mensajes al Parlamento.

\section{Poderes compartidos} diente:

Corresponde al Presidente, con refrendo del órgano correspon-

- Nombrar a los miembros del Gobierno.

- Promulgar las leyes.

- Convocar al Parlamento en sesión extraordinaria.

- Firmar las órdenes y decretos acordados por el Consejo de Ministros.

- Otorgar empleos civiles y militares. 
- Acreditar embajadores.

- Ejercer el Derecho de gracia.

- Nombrar a los miembros del Consejo de la Magistratura.

- Ejercer prerrogativas en materia de defensa nacional así como en materia de política internacional.

Los poderes presidenciales dependen, en el régimen político francés de la coincidencia o no de las mayorías parlamentaria y presidencial en una misma fuerza política, de forma que cuando el Presidente de la República y el Primer Ministro se encuentran apoyados en la misma fuerza política, el primero asume un papel preponderante puesto que la diferencia entre las funciones propias y las compartidas no estarán sometidas a enfrentamiento. Por el contrario, en el caso de discordancia ente las orientaciones políticas presidencial y parlamentaria, se hará preciso la "cohabitación" entre ambos poderes, cohabitación que en Francia se ha producido durante el período parlamentario 1981-1986 y 1993-1995, lo que puede dar lugar la existencia de fuertes fricciones de gobierno. Cuando las fuerzas coinciden en las funciones de gobierno, el Presidente de la República se convierte en una especie de "monarca republicano".

\section{Los Presidentes de la República Francesa}

\begin{tabular}{lccl}
\hline & $\begin{array}{c}\text { Début } \\
\text { du } \\
\text { mandat }\end{array}$ & $\begin{array}{c}\text { Fin } \\
d u \\
\text { mandat }\end{array}$ & \\
\hline $\begin{array}{l}\text { L. N. Bonaparte } \\
\text { (1808-1873) }\end{array}$ & $18-12-1848$ & $3-12-1852$ & $\begin{array}{l}\text { Un an après le "coup d'Etat" du 2 } \\
\text { décembre 1851, la dignité impèriale } \\
\text { est rétablie. (Durée du mandat: } 4 \\
\text { ans) }\end{array}$ \\
\hline
\end{tabular}

A. Thiers

(1797-1877)

17-2-1871 24-5-1873

"Chef du pouvoir exécutif" jusqu'au 31 août 1871, il démissionne après avoir été mis en minorité para la Chambre à qui il refuse de "faire prévaloir dans le gouvernement une politique résolument conservatrice". (Durée du mandat: 2 ans 6 mois)

\begin{tabular}{lll}
\hline $\begin{array}{l}\text { Mac-Mahon } \\
\text { (1808-1893) }\end{array}$ & 24-5-1873 30-1-1879 & $\begin{array}{l}\text { Les élections d'octobre 1877 amè- } \\
\text { nent à la Chambre une majorité ré- } \\
\text { publicaine qui pousse le Président, } \\
\text { jugé trop conservateur, à démis- } \\
\text { sionner. (Durée du mandat: } 5 \text { ans } 8 \\
\text { mois) }\end{array}$
\end{tabular}




\section{Los Presidentes de la República Francesa (Continuación)}

\begin{tabular}{lccl}
\hline & $\begin{array}{c}\text { Début } \\
\text { du } \\
\text { mandat }\end{array}$ & $\begin{array}{c}\text { Fin } \\
\text { du } \\
\text { mandat }\end{array}$ & \\
\hline J. Grévy & $30-1-1879$ & $3-12-1887$ & $\begin{array}{l}\text { Réélu en 1885, il est contraint à } \\
\text { démissionner à la suite du uscanda- } \\
\text { (1807-1891) }\end{array}$ \\
& & $\begin{array}{l}\text { les décorations" dans lequel son } \\
\text { gendre est impliqué. (Durée du } \\
\text { mandat: } 8 \text { ans 10 mois) }\end{array}$ \\
\hline
\end{tabular}

\begin{tabular}{llll}
\hline $\begin{array}{l}\text { S. Carnot } \\
\text { (1837-1894) }\end{array}$ & $3-12-1887$ & $24-6-1894$ & $\begin{array}{l}\text { Assassiné par Casério après avoir re- } \\
\text { fusé la grâce de l'anarchiste Valliant, } \\
\text { auteur d'un attentat à la Chambre. } \\
\text { (Durée du mandat: } 6 \text { ans } 7 \text { mois) }\end{array}$ \\
\hline
\end{tabular}

\begin{tabular}{lccc}
\hline $\begin{array}{l}\text { J. Casimir-Perier } \\
\text { (1847-1907) }\end{array}$ & 24-6-1894 & 15-1-1895 & $\begin{array}{l}\text { Démissionne après les attaques des } \\
\text { députés socialistes sur ses liens } \\
\text { avec le ucomité des forges". (Durée } \\
\text { du mandat: } 6 \text { mois) }\end{array}$ \\
\hline
\end{tabular}

\begin{tabular}{lccl}
\hline $\begin{array}{l}\text { F. Faure } \\
\text { (1841-1899) }\end{array}$ & $17-1-1895$ & $16-2-1899$ & $\begin{array}{l}\text { Décède subitement à Iñ'Elysée. } \\
\text { (Durée du mandat: } 4 \text { ans } 1 \text { mois) }\end{array}$ \\
\hline $\begin{array}{l}\text { E. Loubet } \\
\text { (1838-1929) }\end{array}$ & $18-2-1899$ & $18-2-1906$ & (Durée du mandat: 7 ans) \\
\hline
\end{tabular}

\begin{tabular}{lccl}
\hline $\begin{array}{l}\text { A. Fallières } \\
\text { (1841-1931) }\end{array}$ & $18-2-1906$ & $18-2-1913$ & (Durée du mandat: 7 ans) \\
\hline $\begin{array}{l}\text { R. Poincaré } \\
\text { (1860-1934) }\end{array}$ & $18-2-1913$ & $17-2-1920$ & (Durée du mandat: 7 ans) \\
\hline $\begin{array}{l}\text { P. Deschanel } \\
\text { (1855-1922) }\end{array}$ & $17-2-1920$ & $21-9-1920$ & $\begin{array}{l}\text { Frappé d'incapacité, il est poussé à la } \\
\text { démission. (Durée du mandat: } \\
\text { mois) }\end{array}$ \\
\hline
\end{tabular}

A. Millerand 23-9-1920 11-6-1924 II démissionne après que la Cham-
$(1859-1943)$ bre ait refusé "d'entrer en relations" avec le président du Conseil qu'il a choisi hors du Parlement. (Durée du mandat: 3 ans 8 mois)

\begin{tabular}{lccl}
\hline $\begin{array}{l}\text { G. Doumergue } \\
\text { (1863-1937) }\end{array}$ & $13-6-1924$ & $13-6-1931$ & (Durée du mandat: 7 ans) \\
\hline $\begin{array}{l}\text { P. Doumer } \\
\text { (1857-1932) }\end{array}$ & $13-6-1931$ & $6-5-1932$ & $\begin{array}{l}\text { Assassiné par Gorguloff. (Durée du } \\
\text { mandat: 11 mois) }\end{array}$ \\
\hline $\begin{array}{l}\text { A. Lebrun } \\
\text { (1871-1950) }\end{array}$ & $10-5-1932$ & $13-7-1940$ & $\begin{array}{l}\text { Réélu en 1939, se retire de fait à la } \\
\text { publication de l'Acte constitutionnel } \\
n^{2} 1 \text { du 11 juillet 1940 qui dispose: } \\
\text { "Nous, Philippe Pétain, maréchal de } \\
\text { France, déclarons assumer les fonc- } \\
\text { tions de chef de l'Etat français". }\end{array}$ \\
\hline & & &
\end{tabular}




\section{Los Presidentes de la República Francesa (Continuación)}

\begin{tabular}{|c|c|c|c|}
\hline & $\begin{array}{l}\text { Début } \\
\text { du } \\
\text { mandat }\end{array}$ & $\begin{array}{l}\text { Fin } \\
\text { du } \\
\text { mandat }\end{array}$ & \\
\hline $\begin{array}{l}\text { V. Auriol } \\
(1884-1966)\end{array}$ & $16-1-1947$ & 23-12-1953 & (Durée du mandat: 7 ans) \\
\hline $\begin{array}{l}\text { R. Coty } \\
(1882-1962)\end{array}$ & 23-12-1953 & 8-1-1959 & $\begin{array}{l}\text { Après l'adoption de la Constitution } \\
\text { du } 4 \text { octobre } 1958 \text {, il ne se présente } \\
\text { pas à l'élection présidentielle orga- } \\
\text { nisée en décembre } 1958 \text {. (Durée du } \\
\text { mandat: } 6 \text { ans } 2 \text { mois) }\end{array}$ \\
\hline $\begin{array}{l}\text { Ch. de Gaulle } \\
(1890-1970)\end{array}$ & 8-1-1959 & $28-4-1969$ & $\begin{array}{l}\text { Se retire après l'annonce des résul- } \\
\text { tats du référendum du même jour. } \\
\text { (Durée du mandat: } 10 \text { ans } 3 \text { mois) }\end{array}$ \\
\hline $\begin{array}{l}\text { G. Pompidou } \\
\text { (1911-1974) }\end{array}$ & $19-6-1969$ & 2-4-1974 & $\begin{array}{l}\text { Décès. (Durée du mandat: } 4 \text { ans } 10 \\
\text { mois) }\end{array}$ \\
\hline $\begin{array}{l}\text { V. Giscard d'Estaing } \\
(1926-. .)\end{array}$ & $24-5-1974$ & 21-5-1981 & (Durée du mandat: 7 ans) \\
\hline $\begin{array}{l}\text { F. Miterrand } \\
(1916-. .)\end{array}$ & $21-5-1981$ & & $\begin{array}{l}\text { Réélu en mai 1988. (Durée du pre- } \\
\text { mier mandat: } 7 \text { ans) }\end{array}$ \\
\hline
\end{tabular}

Datos ofrecidos por "Documentos de Estudio" número 1.06. París 1993.

Las Elecciones Presidenciales (1965-1988)

\begin{tabular}{lcccccc}
\hline & \multicolumn{2}{c}{ Premier tour } & \multicolumn{3}{c}{ Second tour } \\
\hline & Suffrages & $\%$ & $\%$ & Suffrages & $\%$ & $\%$ \\
\hline & 5 décembre 1965 & & \multicolumn{3}{c}{ 19 décembre 1965 } \\
Inscrits & 28.913 .422 & 100 & - & 28.902 .704 & 100 & - \\
Votants & 24.502 .957 & 84,7 & - & 24.371 .647 & 84,3 & - \\
Suffrages exprimés & 24.254 .554 & 83,9 & 100 & 23.704 .434 & 82 & 100 \\
Ch. de Gaulle & 10.828 .523 & 37,6 & 44,6 & 13.083 .699 & 45,3 & 52 \\
F. Miterrand & 7.694 .003 & 26,7 & 31,8 & 10.619 .735 & 36,7 & 44,8 \\
J. Lecanuet & 3.777 .119 & 13 & 15,6 & & & \\
J. L. Tixier-Vignancour & 1.260 .208 & 4,3 & 5,2 & & & \\
P. Marcilhacy & 415.018 & 1,4 & 5,2 & & & \\
M. Barbu & 279.683 & 0,9 & 1,1 & & & \\
\hline
\end{tabular}


Las Elecciones Presidenciales (1965-1988) (Continuación)

\begin{tabular}{|c|c|c|c|c|c|c|}
\hline & \multicolumn{3}{|c|}{ Premier tour } & \multicolumn{3}{|c|}{ Second tour } \\
\hline & Suffrages & $\%$ & $\%$ & Suffrages & $\%$ & $\%$ \\
\hline & 1er juin 1969 & & & 15 juin 1969 & & \\
\hline $\begin{array}{l}\text { Inscrits } \\
\text { Votants } \\
\text { Suffrages exprimés } \\
\text { G. Pompidou } \\
\text { A. Poher } \\
\text { J. Duclos } \\
\text { G. Defferre } \\
\text { M. Rocard } \\
\text { L. Ducatel } \\
\text { A. Krivine }\end{array}$ & $\begin{array}{r}29.513 .361 \\
22.899 .034 \\
22.603 .998 \\
10.051 .816 \\
5.268 .651 \\
4.808 .285 \\
1.133 .222 \\
816.471 \\
286.447 \\
239.106\end{array}$ & $\begin{array}{l}100 \\
77,6 \\
76,6 \\
34,1 \\
17,8 \\
16,3 \\
3,8 \\
2,8 \\
1 \\
0,8\end{array}$ & $\begin{array}{c}- \\
\overline{100} \\
44,5 \\
23,3 \\
21,3 \\
3,6 \\
3,6 \\
1,3 \\
1\end{array}$ & $\begin{array}{r}29.500 .334 \\
20.311 .287 \\
19.007 .489 \\
11.064 .371 \\
7.943 .118\end{array}$ & $\begin{array}{c}100 \\
68,9 \\
64,5 \\
37,5 \\
27\end{array}$ & $\begin{array}{c}- \\
\overline{100} \\
58,2 \\
41,8\end{array}$ \\
\hline A. Krivine & 5 mai 1974 & & & 19 mai 1974 & & \\
\hline $\begin{array}{l}\text { Inscrits } \\
\text { Votants } \\
\text { Suffrages exprimés } \\
\text { F. Mitterrand } \\
\text { V. Giscard d'Estaing } \\
\text { J. Chaban-Delmas } \\
\text { J. Royer } \\
\text { A. Laguille } \\
\text { R. Dumont } \\
\text { J. M. Le Pen } \\
\text { E. Muller } \\
\text { A. Krivine } \\
\text { B. Renouvin } \\
\text { J. C. Sebag } \\
\text { G. Héraud }\end{array}$ & $\begin{array}{r}30.602 .953 \\
25.775 .743 \\
25.538 .636 \\
11.055 .373 \\
8.326 .774 \\
3.857 .728 \\
810.540 \\
595.247 \\
337.800 \\
190.921 \\
176.279 \\
93.990 \\
43.722 \\
42.007 \\
19.255\end{array}$ & $\begin{array}{l}100 \\
84,2 \\
83,4 \\
36,1 \\
27,2 \\
12,6 \\
2,7 \\
1,9 \\
1,1 \\
0,6 \\
0,6 \\
0,3 \\
0,1 \\
0,1 \\
0,1\end{array}$ & $\begin{array}{l}- \\
-\overline{100} \\
43,2 \\
32,6 \\
15,1 \\
3,2 \\
2,3 \\
1,3 \\
0,7 \\
0,7 \\
0,4 \\
0,2 \\
0,2 \\
0,1\end{array}$ & $\begin{array}{l}30.600 .775 \\
26.724 .595 \\
26.367 .807 \\
12.971 .604 \\
13.396 .203\end{array}$ & $\begin{array}{l}100 \\
87,3 \\
86,1 \\
42,4 \\
43,7\end{array}$ & $\begin{array}{c}- \\
\overline{100} \\
49,2 \\
50,8\end{array}$ \\
\hline G. Héraud & 26 avril 1981 & & & 10 mai 1981 & & \\
\hline $\begin{array}{l}\text { Inscrits } \\
\text { Votants } \\
\text { Suffrages exprimés } \\
\text { V. Giscard d'Estaing } \\
\text { F. Mitterrand } \\
\text { J. Chirac } \\
\text { G. Marchais } \\
\text { B. Lalonde } \\
\text { A. Laguiller } \\
\text { M. Crépeau } \\
\text { M. Debré } \\
\text { M. F. Garaud } \\
\text { H. Bouchardeau }\end{array}$ & $\begin{array}{r}36.398 .859 \\
29.516 .082 \\
29.038 .117 \\
8.222 .432 \\
7.505 .960 \\
5.225 .848 \\
4.456 .922 \\
1.126 .254 \\
668.057 \\
642.847 \\
481.821 \\
386.623 \\
321.353\end{array}$ & $\begin{array}{l}100 \\
81,1 \\
79,8 \\
22,6 \\
20,6 \\
14,4 \\
12,2 \\
3,1 \\
1,8 \\
1,8 \\
1,3 \\
1,1 \\
0,9\end{array}$ & $\begin{array}{c}- \\
\overline{1} \\
100 \\
28,5 \\
25,9 \\
18 \\
15,3 \\
3,9 \\
2,3 \\
2,2 \\
1,7 \\
1,3 \\
1,1\end{array}$ & $\begin{array}{l}36.398 .762 \\
31.249 .552 \\
30.350 .568 \\
14.642 .306 \\
15.708 .262\end{array}$ & $\begin{array}{l}100 \\
85,9 \\
83,4 \\
40,3 \\
43,1\end{array}$ & $\begin{array}{l}- \\
- \\
100 \\
48,2 \\
51,8\end{array}$ \\
\hline
\end{tabular}




\section{Las Elecciones Presidenciales (1965-1988) (Continuación)}

\begin{tabular}{lcccccc}
\hline & \multicolumn{2}{c}{ Premier tour } & \multicolumn{3}{c}{ Second tour } \\
\hline & Suffrages & $\%$ & $\%$ & Suffrages & $\%$ & $\%$ \\
\hline 24 avril 1988 & & & $\mathbf{8}$ mai $\mathbf{1 9 8 8}$ & & \\
Inscrits & 38.179 .118 & 100 & - & 38.168 .869 & 100 & - \\
Votants & 31.059 .300 & 81,3 & - & 32.085 .071 & 84,1 & - \\
Suffrages exprimés & 30.436 .744 & 79,7 & 100 & 30.923 .249 & 81 & 100 \\
F. Mitterrand & 10.381 .332 & 27,2 & 34,1 & 16.704 .279 & 43,8 & 54 \\
J. Chirac & 6.075 .160 & 15,9 & 19,9 & 1.218 .970 & 37,2 & 46 \\
R. Barre & 5.035 .144 & 13,2 & 16,5 & & & \\
J. M. Le Pen & 4.376 .742 & 11,4 & 14,4 & & & \\
A. Lajoinie & 2.056 .261 & 5,4 & 6,8 & & & \\
A. Waechter & 1.149 .897 & 3 & 3,8 & & & \\
P. Juquin & 639.133 & 1,7 & 2,1 & & & \\
A. Laguiller & 606.201 & 1,6 & 2 & & & \\
P. Boussel & 116.874 & 0,3 & 0,4 & & & \\
\hline
\end{tabular}

Datos ofrecidos por «Documentos de Estudio» número 1.06. París 1993.

\section{LA CAMPAÑA PRESIDENCIAL DE 1995}

El 23 de abril y, en principio, el 7 de mayo, los franceses van a proceder a la elección del Presidente de la República. La campaña comienza oficialmente el 7 de abril, dieciseis días antes de la primera vuelta electoral, debiendo terminar veinticuatro horas antes de la votación.

La instauración del sistema de 2 vueltas, posibilita que a la primera concurra un gran número de candidatos apoyados por sus respectivas fuerzas que, aunque tengan conciencia de la imposibilidad de obtener uno de los dos primeros puestos, lo que les permitiría a optar al cargo de Presidente, sí permite que cada fuerza política pueda constatar los votos populares y el apoyo con el que cuentan, lo que puede tener una especial relevancia para negociar, apoyar y adoptar acuerdos de cara a la segunda vuelta ${ }^{7}$.

7 Núñez Rivero, C.: Opus cit, pág. 521. 
Como ha indicado Touraine ${ }^{8}$, "la campaña presidencial en Francia no es un acontecimiento político sino un juego de sociedad". Un cierto número de candidatos que no tienen ninguna posibilidad de triunfar tratan de influir en los que pueden ganar.

Para esta campaña, algo más de 40 millones de electores franceses están convocados a las urnas para seleccionar, de entre nueve candidatos, los dos finalistas entre los que se elegirán el 7 de mayo, caso de que, como es de preveer, sea necesaria una segunda vuelta, al sucesor de François Mitterrand.

Al margen de los enfrentamientos personales entre candidatos, la campaña no ha sido esclarecedora, sino todo lo contrario, conforme han hablado los candidatos ha ido aumentando el número de indecisos, que a una semana de la primera vuelta alcanza un 38 por 100 de votantes, frente al 33 por 100 que manifiestan su indicisión dos semanas antes ${ }^{9}$.

Jacques Chirac parte siempre como posible triunfador en la primera y segunda vuelta. Balladur y Jospin se disputan el segundo puesto en una campaña que anuncia la consolidación de la extrema derecha, una subida de la extrema izquierda, el fracaso del socialismo y la insignificación de los ecologistas.

A pesar de que son varias las formaciones políticas que concurren a las elecciones presidenciales, la intención de voto, según muestran los sondeos electorales, parece decantarse por dos partidos políticos, cuyos candidatos pueden optar a la segunda vuelta electoral:

\section{Reagrupamiento para la República (RPR)}

Aun cuando es un partido de reciente creación, ya que oficialmente nace del 5 de diciembre de 1976, no es más que una especie de reencarnación de la fuerza política que inspiró al General de Gaulle hace más de treinta años.

Esta formación política, que presenta a dos candidatos presidenciales, Chirac y Balladur, mantiene vigente las directrices básicas del gaullismo, a saber, la independencia de Francia respecto del Este y del Oeste, una defensa nacional independiente que incluya una fuerza disuasiva nu-

1995.

8 Touraine, A.: "La Francia de después de Mitterrand» El País, 17 de abril de

9 Datos ofrecidos por Le Monde. 16 de abril de 1995. 
clear en el marco de la OTAN, la fe en la solidez de las instituciones de la V. República y una fuerte oposición al comunismo.

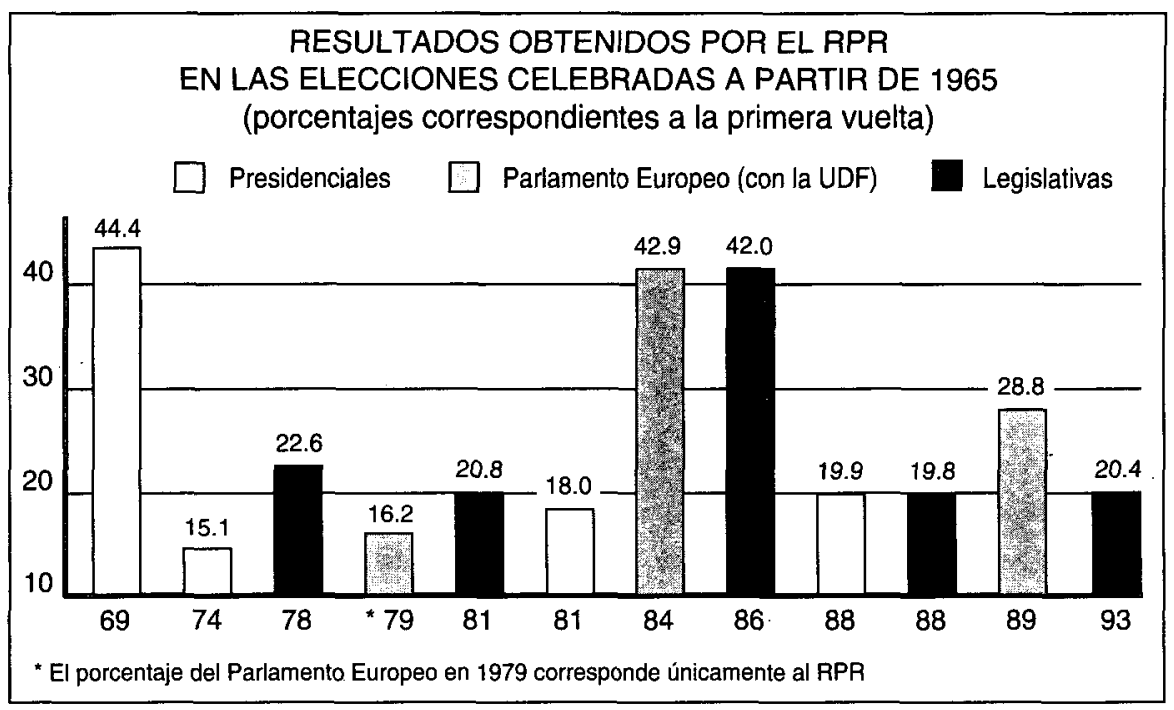

Datos ofrecidos por la Embajada de Francia en España.

\section{El Partido Socialista}

Aunque las raíces del socialismo francés son muy antiguas, la actual ideología socialista hace hincapié en la construcción de la democracia social no solo mediante una economía planificada sino también por medio de la independencia nacional, pero dentro de un marco europeista. En el actual PS subsisten varios matices de la teoría socialista que van desde los moderados de Rocard a la tendencia más de izquierda de Chevenment, lo que ha supuesto una dura tarea de elección de candidato presidencial, ante la negativa de Delors de presentarse, que se ha saldado con la candidatura de Jospin.

Miterrand, que ha concluido 14 años de mandato presidencial, abandona la presidencia en el marco de un partido agotado por el ejercicio del poder, acusado de graves problemas de corrupción y con su fuerza política muy disminuída en la Asamblea Nacional. 


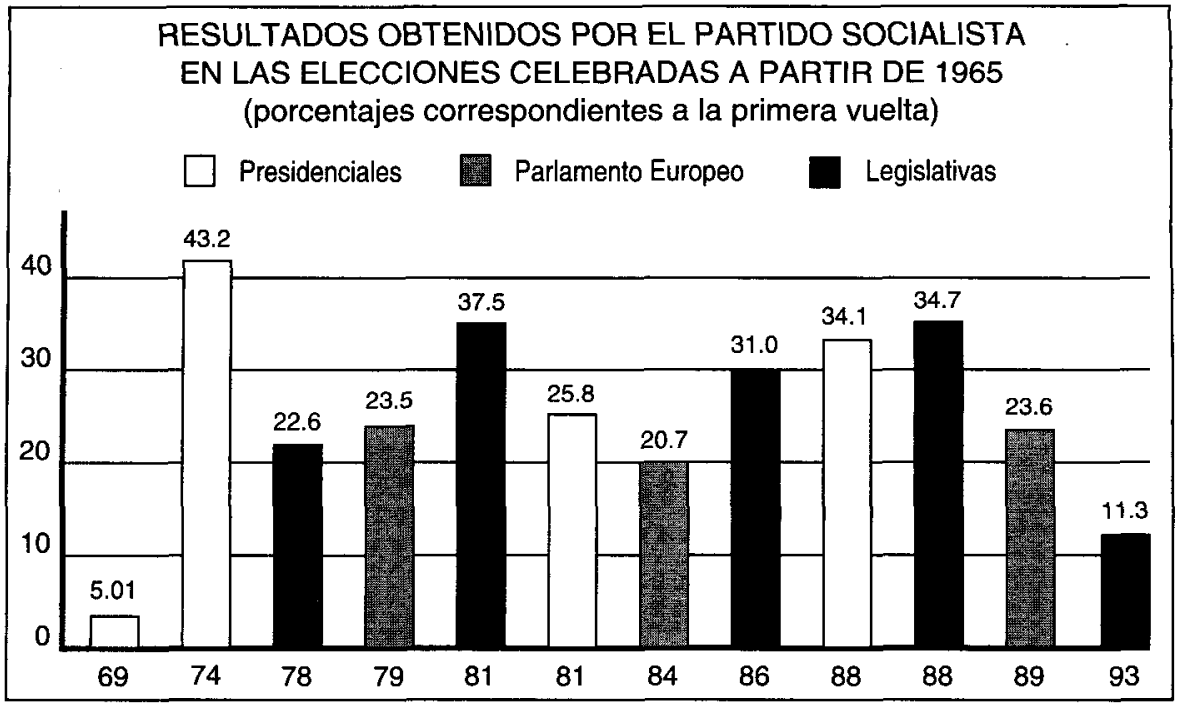

Datos ofrecidos por la Embajada de Francia en España.

Los principales candidatos a la elección presidencial son:

- Jacques Chirac ................... Reagrupación para la República

- Edouard Balladur ................ Reagrupación para la República

- Lionel Jospin ....................... Partido Socialista

- Jean-Marie Le Pen .............. Frente Nacional

- Robert Hue .......................... Partido Comunista

- Philippe de Viliers ............... Movimiento por Francia

- Arlette Laguilier

- Dominique Voynet Lucha Obrera

- Jaques Cheminade ............. Nueva solidaridad

\section{Los Candidatos Presidenciales en Campaña}

Las pretensiones de los canditados a ocupar la Presidencia de la República francesa son:

JACQUES CHIRAC (Reagrupación para la República)

Este discípulo de Pompidou, que lleva batallando más de veinte años por la presidencia, parte como favorito para ganar las elecciones 
presidenciales en Francia. Rupturista, Chirac habrá de ofrecer la ruptura con dos años de supuesto inmovilismo conservador en el Gobierno, así como luchar contra Balladur, candidato por el mismo partido, por evitar la división del voto agrupacionista, aunque es Chirac quien cuenta con el apoyo del aparato del partido ${ }^{10}$.

Este eminente político que se ha aliado y combatido con casi todas las fuerzas políticas francesas ${ }^{11}$, lanza una serie de mensajes que han gustado a los franceses, alarmados por la creciente crisis social, aunque carentes de concreción, entre los que se encuentran el cambio, el empleo, el pacto republicano, la lucha contra la marginación y la solidaridad.

Si supera el 25 por 100 de votos en la primera vuelta tendrá a su favor la aritmética; si el rival es Jospin, tendrá a su favor la lógica política; si no llega al 25 por 100 y se enfrenta a Balladur, todo estará en el aire ${ }^{12}$.

\section{EDOUARD BALLADUR (Reagrupación para la República)}

El Primer Ministro, que en un primer momento ha obtenido un gran respaldo popular, trata de recuperar el terreno perdido por su candidatura ante sus rivales, e intenta conseguir entre los indecisos el 5 por 100 de los votos que necesita para su llegada a la segunda vuelta ${ }^{13}$, para lo que no ha dudado en ningún momento en criticar a Chirac con graves acusaciones, en un período en que los sondeos de opinión estrechan las diferencias entre los dos candidatos gaullistas.

Más que un programa, lo que Balladur ofrece es su método de gobierno, ya ensallado en los dos últimos años de mandato, que en los últimos tiempos se ha visto salpicado por una serie de escándalos de corrupción. Bajo el lema de "El cambio con seguridad», el candidato presenta como emblemas de su quehacer: la estabilidad del franco, la creación de empleo a través de la recuperación económica y la construcción europea ${ }^{14}$.

Balladur tiene a su favor el voto de los mercados financieros internacionales, aunque no confían en su victoria. El Primer Ministro francés se ha propuesto dar el empujón a los valores que han marcado su gestión desde 1993, intentando comunicar a los franceses la estabilidad y la cal-

10 El País, 20 de abril de 1995.

11 Fue antiliberal en 1981, y ultraliberal en 1988.

12 EnRIC GonzÁlez. "¿Chirac Presidente?» El País, 23 de abril de 1995.

$13 \quad \mathrm{ABC}, 21$ de abril de 1995.

14 El País, 23 de abril de 1995. 
ma como mecanismos necesarios para el desarrollo de una adecuada acción de Gobierno.

\section{LIONEL JOSPIN (Partido Socialista francés)}

Aunque las raíces del socialismo francés se remontan a los teóricos utópicos de principios del siglo XIX, en la actualidad, y tras haber perdido la Presidencia del Gobierno, el PSF puede sufrir una gran derrota como consecuencia de la crisis social y económica que sufre Francia, y por los casos de corrupción en que se ha visto involucrado el socialismo. La retirada de Mitterrand y la negativa de Jacques Delors a encabezar la carrera presidencial, han puesto en un aprieto al socialismo que, no obstante, ha sabido elegir a un buen candidato - Lionel Jospin-.

A pesar del enfrentamiento de los barones socialistas, la tenacidad de Jospin, que no ofrece utopías, ni promete paraísos, ha podido alzarse con el mérito de convertirse en la única esperanza creíble de la izquierda ${ }^{15}$. La influencia del clan Delors se hace notar en el programa de Jospin que ha tenido muy dificil proponer una idea de cambio y al mismo tiempo respetar globalmente la herencia de Mitterrand ${ }^{16}$.

Jospin, que representa el socialismo "serio" ${ }^{17}$, propone un programa de creación de empleo y reconstrucción de los sectores sociales más desprotegidos. Postula una "nueva práctica del poder" mediante un "presidente-ciudadano", para la moralización de la vida pública. Relanza las ideas de Delors sobre la Unión Europea basada en cuatro pilares: una moneda única; un programa único de creación de empleo; una política comercial europea y un sistema de defensa basado en un ejército europeo.

En el aspecto institucional, destaca en el programa de Jospin, el anuncio de intención de reducción del mandato presidencial de 7 a 5 años y su deseo, para el supuesto de triunfo, de disolución de la Asamblea Nacional para evitar, en la medida de lo posible, la actual cohabitación politica.

\section{JEAN MARIE LE PEN (Frente Nacional)}

La sorpresa de las elecciones europeas de 1984, en las que Le Pen superó el 10 por 100 de los votos, y el 14 por 100 de votos que obtiene en las elecciones presidenciales de 1988 se han convertido en una auténtica

\footnotetext{
El Pais, 22 de abril de 1995.

El País, 23 de abril de 1995.

ABC, 23 de abril de 1995.
} 
preocupación en el Estado francés que ve, impasible, el ascenso de las posturas más extremistas de la derecha.

Le Pen es el más feroz de la Partida ${ }^{18}$. Patriotista en extremo, su campaña ha contado con la brutalidad habitual ${ }^{19}$. Tiene soluciones para todo: propone la expulsión de tres millones de inmigrantes para la eliminación del paro; el restablecimiento de la pena de muerte y el reforzamiento de la policía para la superación de los problemas sociales.

Antieropeista por excelencia, denuncia el Tratado de Maastrich, y en el terreno económico defiende la supresión del impuesto sobre la renta, la denuncia del GATT y un riguroso proteccionismo económico.

Su radicalismo hace de Le Pen un aliado indeseable para las fuerzas de centro-derecha, que podrian arriesgar el voto del electorado moderado en caso de acercamientos explícitos.

Su principal atractivo consiste en que permite a los sectores más criticos con la actuación gubernamental la oportunidad de desviar contra culpables claramente identificables "sus frustaciones políticas y económicas»"

\section{ROBERT HUE (Partido Comunista)}

Este nuevo rostro del Partido Comunista, sucesor de Marchais, intenta rejuvenecer el comunismo. Las elecciones presidenciales constituyen la oportunidad de romper el pasado estalinista del partido al que representa, e intentar poner fin a su largo declive. Se ha desmarcado de algunas costumbres en el ejercicio del poder, hablando de un comunismo nuevo que no tiene nada que ver con el comunismo del Este.

Su programa gira, desde el punto de vista internacional, en torno al rechazo al Tratado de Maastrich, proponiendo, en el plano económico, la subida salarial y la oposición a la austeridad económica, con una masiva distribución de ayuda a los más pobres y necesitados que saldría de los beneficios obtenidos por los sectores más favorecidos.

EI PC afirma su vocación de participar en todos los niveles en las instituciones francesas, incluso el Gobierno, pero su posición antimaastrich hace casi imposible su unión a un posible gobierno socialista que respalda ampliamente los compromisos europeos.

${ }^{20}$ El País. Opinión. 26 de abril de 1995. 


\section{PHILIPPE DE VILLIERS (Movimiento por Francia)}

Los franceses que se sitúan a la derecha del RPR dudan entre la actitud "vociferante" ${ }^{21}$ de Le Pen y la cruzada iniciada por De Villiers.

Todo indica que será más difícil que repita los resultados de las elecciones al Parlamento europeo donde se hizo con el 12,4 por 100 de los votos y 13 escaños.

Representa De Villiers a la derecha más tradicionalista. Antieuropeísta y católico exaltado, en la campaña se ha presentado como uazote de la corrupción " ${ }^{22}$ y dique contra el poder que alcanza la Comisión europea. Cuatro son los puntos que centran su campaña política: rechazo global a Maastrich, proteccionismo comercial, supresión del impuesto sobre la renta y fuerte control sobre la inmigración, aunque en este último aspecto, frente al xenofobismo de Le Pen, muestra una preferencia por la inmigración comunitaria.

Este candidato es el gran defensor de la "soberanía nacional", acepta el servicio público, pero se muestro favorable a una revisión que abarque, incluso, lo establecido por la Revolución francesa ${ }^{23}$.

\section{ARLETTE LAGUILLER (Lucha Obrera)}

Esta veterana candidata a las elecciones presidenciales francesas, se ha presentado en 1974, 1979, 1988 y 1995, presenta un programa poco desarrollado y repetido hasta la saciedad. Sus puntos principales se manifiestan en una doble vertiente: en el aspecto económico, defiende la incautación de toda empresa que recurra a los despidos, así como un impuesto del 50 por 100 sobre los beneficios empresariales. En el aspecto social su programa parte de un aumento salarial lineal y la reducción de la jornada laboral a 35 horas, junto al establecimiento de un programa estatal que mejore la sanidad y el trasporte público.

No obstante, los aspectos más significativos de su discurso se centran en la crítica que realiza al resto de formaciones políticas que concurren a las elecciones presidenciales ${ }^{24}$.

\footnotetext{
$21 \quad$ ABC. 23 de abril de 1995.

$22 \quad$ El País. 23 de abril de 1995.

23 El País. 20 de abril de 1995.

24 "Los comunistas, afirman la candidata, están vendidos al capitalismo.
} Los socialistas son la reencarnación del diablo con rabo. A la derecha hay que meterla en las calderas de Pedro Botero". ABC. 23 de abril de 1995. 
DOMINMIQUE VOYONET (Verdes)

La candidatura ecologista a la Presidencia francesa tiene a su favor haber conseguido romper con los anteriores líderes verdes, Lalonde y Waechter, que produjeron un grave deterioro en la lucha por la ecología.

Luchadora contra el llamado "voto útil", ha anunciado, no obstante, que pedirá su voto en favor de Jospin en el supuesto de que llegue a una segunda vuelta, puesto que se considera como un representante de una fuerza minoritaria pero imprescindible para cualquier proyecto gubernamental de la izquierda.

Propone un aumento de impuestos para las rentas más altas y para los contaminantes y una reducción de la jornada laboral de 35 horas. Propugna la solidaridad internacional y la prohibición de las armas nucleares. En el aspecto institucional defiende un mayor poder para el Parlamento.

En su campaña ha intentado unir tres corrientes: la ecología, el feminismo y el izquierdismo.

\section{JACQUES CHEMINADE (Nueva Solidaridad)}

Bajo el lema "Francia para reconstruir el Mundo" se presenta este gran desconocido de la política francesa que pretende que Francia asuma un liderazgo mundial.

Cheminade ataca el capitalismo especulativo y el liberalismo salvaje y culpa al mundo financiero de ser el culpable de la crisis mundial, a la vez que pretende reivindicar la figura del troskista La Rouche -eterno candidato perdedor a la presidencia de EE.UU. ${ }^{25}$.

Este "pintoresco" candidato, que se apoya en el budismo y el espiritismo para hacer campaña, parte con el peor porcentaje en los sondeos electorales a la Presidencia francesa.

\section{Sondeos Electorales}

Significativos, no tanto en lo que a resultados electorales respecta, sino en lo relativo a las conclusiones que de ellos pueden obtenerse, son los sondeos electorales que una semana antes de la jornada electoral se hacen públicos en Francia. 
Frente al 36 por 100 de intención de voto que obtiene Balladur en los primeros sondeos electorales ${ }^{26}$, la última encuesta ${ }^{27}$ le otorga un 19 por 100, caida que se corresponde inversamente con la ascensión de voto que ostenta el segundo candidato del RPR, Chirac, que pasa del 19 por 100 al 24 por 100.

El tercer candidato en liza - Jospin-pasa del 14 por 100 al 19,5 por $100^{28}$, superando en espectativas a Balladur, algo impensable cuando fue anunciada su candidatura al frente del Partido Socialista francés, y que, en gran medida, se debe, de un lado al enfrentamiento de los dos candidatos de centro-derecha, y de otro a su capacidad para reagrupar y dar homogeneidad a un proyecto socialista, aunque se determine que el Primer Ministro tendía más posibilidades que el candidato socialista en una segunda vuelta ${ }^{29}$.

\section{Evolución de la intención de voto a los tres principales candidatos}

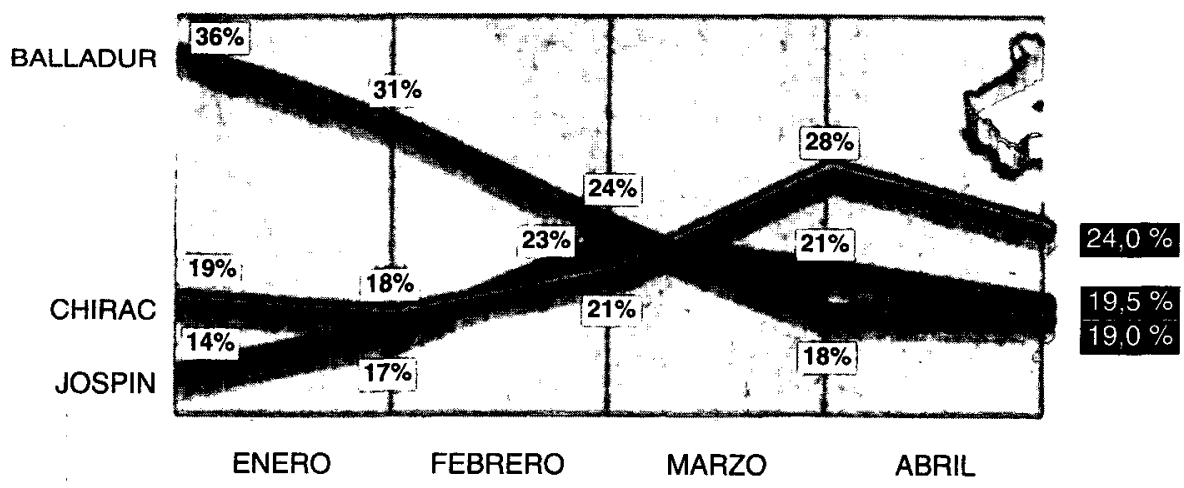

Uno de los aspectos más controvertidos y preocupantes, viene determinado por el ascenso obtenido por Le Pen que, de obtener el 14 por 100 de votos que le auguran las encuestas, pasará a convertirse en el cuarto candidato, a tan solo 5 puntos de Balladur, pero teniendo en cuenta que la tendencia de ambos candidatos son inversas, el primero en alza y el se-

26 Le Monde. 18 de enero de 1995.

27 Le Monde. 16 de abril de 1995.

${ }_{28} \quad$ El punto más alto en las encuestas electorales las obtiene en el sondeo publicado por París Match el 5 de marzo de 1995, que le otorga un 23 por 100.

${ }_{29}$ El Mundo. 18 de abril de 1995. 
gundo a la baja, se puede esperar que el líder ultraderechista pueda obtener el tercer puesto.

Los comunistas, que fueron la primera formación de izquierdas hasta que Mitterrand refundió el actual PS ${ }^{30}$, aspiran a frenar su largo declive y a competir en representatividad con el Partido Socialista, si obtiene el 9,5 por 100 de votos que vaticinan los sondeos.

Los otros cuatro candidatos, por separado, a penas cuentan: el nacionalista De Villiers obtenía el 6 por 100; el troskismo el 5 por 100; el ecologismo el 2,5 por 100 y Cheminade el 0,4 por 100.

Sin embargo, los análisis electorales ponen de manifiesto las siguientes conclusiones:

- El 64 por 100 de los franceses desea como Presidente "un aténtico jefe que ponga orden $y$ mando ${ }^{31}$.

- El electorado francés se define inequívocamente hacia la derecha. Los conservadores suman más de un 60 por 100 de intenciones de voto.

- El malestar respecto al sistema da impulso a las candidaturas más contestatarias que, en conjunto, podían alcanzar un 30,5 por 100. Nunca las formaciones tradicionalmente residuales han afrontado una primera vuelta con tan buenas espectativas.

- El candidato socialista Jospin sólo logra convencer a dos tercios de los votantes que se declaran socialistas.

- Los jóvenes respaldarían mayoritariamente a Chirac, mientras que los mayores de 50 años están con Balladur ${ }^{32}$.

- El número de indecisos, a una semana de la primera vuelta, ronda el 35 por 100.

- El malestar político que existe en Francia determina, de cumplirse las espectativas de sondeo ${ }^{33}$, que los dos candidatos que superen la primera vuelta lo harán con menos de la mitad de los votos. 


\section{Resultados Electorales en la primera vuelta}

Los sondeos electorales se han vuelto a equivocar, saltando la sorpresa en la primera vuelta de las presidenciales francesas. De todos los resultados posibles en estas elecciones, el resultado del día 23 de abril es el más polémico porque deja la sucesión de Mitterrand abierta a cualquier hipótesis.

El "ballotage» está servido. Lo que en un principio parecía una fácil victoria de Jacques Chirac, ha terminado por convertirse, casi, en una derrota a manos del candidato socialista Jospin por quien nadie apostaba para el paso a la segunda vuelta ${ }^{34}$.

Con cerca del 24 por 100 de los votos, Jospin puede estar sorprendido de su éxito en movilizar el voto de izquierdas, en fuga y desánimo por los últimos acontencimientos que han sacudido al socialismo, no obstante, el gran sorprendido puede ser Chirac que se ha visto relegado a un segundo puesto. Balladur, que hace unos meses era el favorito a suceder a Mitterrand, queda fuera de la segunda ronda, aunque a escasa distancia de Chirac.

El enfrentamiento a la segunda vuelta no será, por tanto, entre los dos candidatos del RPR, como habían pronosticado los sondeos, aunque Chirac sigue partiendo como favorito.

La victoria de Jospin ha cogido por sorpresa a todo el país, puesto que, aunque su ventaja sobre Chirac no es excesiva, hace pensar que psicológicamente ha ganado las elecciones. Su resultado recuerda mucho al obtenido en 1981 por Mitterrand.

Desde la reelección de Mitterrand en 1988, el PS no levantaba cabeza, no obstante, el hecho de que su actual candidato concurra a la segunda vuelta servirá a Jospin para preservar al PS como pieza clave de la izquierda francesa. 


\section{Resultados de las Elecciones Presidenciales de 1988}

\begin{tabular}{lcccc}
\hline & \multicolumn{2}{c}{ Primera vuelta } & \multicolumn{2}{c}{ Segunda vuelta } \\
\hline Miterrand & 10.381 .332 & $34,10 \%$ & 16.704 .279 & $54,02 \%$ \\
\hline Chirac & 6.075 .160 & $19,96 \%$ & 14.218 .790 & $45,98 \%$ \\
\hline Barre & 5.035 .144 & $16,54 \%$ & & \\
\hline Le Pen & 4.376 .742 & $14,38 \%$ & & \\
\hline Lajoinie & 2.056 .261 & $6,75 \%$ & & \\
\hline Waetcher & 1.149 .897 & $3,77 \%$ & & \\
\hline Juquin & 639.133 & $2,10 \%$ & & \\
\hline
\end{tabular}

Datos oficiales ofrecidos por la Embajada de Francia en España.

Total de inscritos 38.168 .869

Total de votantes 32.085 .071

Votos blancos o nulos 1.161 .822

Total de votos manifestados 30.923 .249

Abstención $15,93 \%$

Resultados de las Elecciones Presidenciales de 1995

\begin{tabular}{lcc}
\hline & \multicolumn{2}{c}{ Primera vuelta } \\
\hline Jospin & 7.101 .992 & $23,30 \%$ \\
\hline Chirac & 6.351 .672 & $20,84 \%$ \\
\hline Balladur & 5.662 .116 & $18,58 \%$ \\
\hline Le Pen & 4.573 .202 & $15,00 \%$ \\
\hline Hue & 2.634 .187 & $8,64 \%$ \\
\hline Laguiller & 1.616 .546 & $5,30 \%$ \\
\hline De Villiers & 1.444 .053 & $4,74 \%$ \\
\hline Voynet & 1.001 .373 & $3,32 \%$ \\
\hline Cheminade & 85.070 & $0,28 \%$ \\
\hline
\end{tabular}

Datos oficiales ofrecidos por el Ministerio del Interior francés.

Total de inscritos

Total de votantes

Votos blancos o nulos

Total de votos manifestados

Abstención 
Jospin ha tenido el mérito de ser el único candidato que presentaba un programa innovador en lo social y muy reformista en lo político 35 que no ha hecho vanas promesas, con lo que ha puesto de manifiesto una cierta coherencia política que ha sido premiada por el electorado. Además ha logrado unir al PS que todavía arrastra la espectacular derrota que sufrió en las legislativas de hace dos años ${ }^{36}$. El triunfo de Jospin es pues un triunfo personal que le permite asumir el liderazgo del socialismo francés.

Tal vez el electorado de izquierdas haya optado por el voto útil, puesto que, aunque los electores socialistas no hayan estado plenamente satisfechos, al menos en un principio, con el candidato, han querido asegurarse la presencia socialista en una segunda vuelta.

Frente al éxito socialista, el gran derrotado en esta primera vuelta ha sido el RPR que ha visto como su Primer Ministro era relegado al tercer puesto, y cómo su principal candidato Jacques Chirac ha sido derrotado por un candidato socialista con poco arraigo personal. El voto de protesta jugó en contra de Chirac y Balladur enfrentado en una "pelea entre jefes" ${ }^{37}$.

No obstante el triunfo socialista, los resultados ofrecen pocas perspectivas de cara que su candidato pueda convertirse en Presidente de la República, puesto que, globalmente, la izquierda suma un 40 por 100 frente al 60 por 100 que roza la derecha.

En la derecha no hubo voto útil, y el voto de protesta, que perjudicó a los jefes gaullistas, benefició a Le Pen, el líder ultraderechista del Frente Nacional que ha obtenido el 15 por 100 de los sufragios, agrupando unos votos que pertenecen a sectores que hasta hace unos años constituían la principal reserva de los votos de la izquierda: parados, jóvenes y obreros no cualificados constituyan más del 50 por 100 de sus votantes ${ }^{38}$. La cota de voto alcanzada por Le Pen le sitúa en una posición importante, y preocupante, de cara a la segunda vuelta.

Lo más espectacular de la primera vuelta a las presidenciales francesas ha sido el ascenso de las fuerzas antisistema, entre ellas sobre todo Le Pen, que alcanza un voto acumulado de casi el 30 por $100^{39}$.

36 Para ver los resultados de las elecciones parlamentarias francesas vease Fernández-Miranda Campoamor, C.: "Revista de Derecho Político no 38",

${ }_{37}$ El País. 24 de abril de 1995.

38 Según una encuesta publicada oir Le Monde el día 25 de abril de 1995.

39 El País. "La amenaza ultra en Francia», 26 de abril de 1995. 
Hue ha mejorado la posición de Lajoinie $(6,7$ por 100) en las elecciones de 1988, pero los comunistas siguien siendo una fuerza minoritaria que tardará mucho tiempo en recuperarse. Sorprende el éxito del troskismo, que de la mano de Laguiller consigue el 5 por 100 de los votos, lo que demuestra el atractivo electoral que ejercen personajes alejados del prototipo de político convencional ${ }^{40}$, con lo que consigue doblar su influencia electoral. Los resultados obtenidos por De Villiers $(4,74$ por 100$)$ le convierten en un punto importante para la segunda vuelta. El 3,3 por 100 de los votos que otiene Voynet ${ }^{41}$ ratifica el retroceso de los verdes y la incapacidad de los ecologistas por articularse como una fuerza política "seria $y$ autónoma»" ${ }^{42}$ en Francia.

Por último, los resultados obtenidos por Cheminade $(0,28$ por 100), ponen en evidencia la credibilidad del político, su campaña y el programa bajo el que se presentaba.

No podemos tampoco olvidar la abstención, más del 20 por 100 , que, aunque se mantiene en el nivel normal de las últimas convocatorias, si supone un crecimiento respecto de la abstención habitual en las elecciones presidenciales en Francia, lo que pone de manifiesto, entre otras causas, el incremento del desinterés que los ciudadanos manifiestan en los aspectos públicos.

July ${ }^{43}$ ha indicado que los resultados electorales muestran cuatro verdades:

- La extrema dispersión de los votos que se ha articulado en cinco sentidos: el voto contestatario de la izquierda (Hue, Laguiller y Voynet), el voto socialista, las dos derechas parlamentarias y por último la extrema derecha (Le Pen) y de la derecha extrema (De Villiers).

- Dos derechas casi empatadas de forma que el debate no ha zanjado el debate de la derecha.

- El ascenso de la izquierda contra los pronósticos que auguraban un importante declive.

\section{- Una presidencia frágil.} 1995.

$40 \quad$ El Mundo. 24 de abril de 1995.

${ }^{41}$ No logra superar el 3,8 por 100 obtenido por WAECHTER en 1988.

42 Rojo, A.: "Los socialistas salvan los muebles». El Mundo, 24 de abril de

43 Serge July es director del diario francés Liberation, hace un análisis de los resultados electorales bajo el título "Francia cortada en cinco", publicado por EI Mundo, 25 de abril de 1995. 
A estas lecturas habría que añadir un aumento espectacular de las fuerzas periféricas, que suman más del 30 por 100 , y el enorme ascenso de las ideas que se manifiestan contrarias a la Unión Europea y a Maastricht ${ }^{44}$.

\section{LA SEGUNDA VUELTA EN LAS ELECCIONES PRESIDENCIALES DE 1995}

\section{Las consecuencias del «Ballotage»}

Los resultados electorales de la primera vuelta anteriormente examinados ponen de manifiesto la capacidad del líder socialista para hacer pasar el mensaje de ruptura dulce con el mitterranismo, así como para convertirse en la figura capaz de reagrupar el voto de la izquierda. No obstante, los resultados obtenidos por Jospin, junto a la suma del voto de las fuerzas de izquierda no son suficientes para asegurarle el triunfo en la segunda vuelta, lo que determina que, sin abandonar su discurso socialdemócrata y europeista, destinado a profundizar las divisiones de la derecha ${ }^{45}$, se haga necesario la búsqueda de votos en el electorado centrista.

La posición de Chirac parece más cómoda, puesto que el reagrupamiento de la derecha parece ser la hipótesis más natural. Sin embargo, no se puede garantizar que en el "ballotage" todo el centro-derecha y la derecha vayan a votar a Chirac, lo que determina que la campaña chiraquiana se vea envuelta en un profundo entramado de negociaciones.

Jospin ha hecho un llamamiento "por las nuevas ideas y el progreso de toda la izquierdan ${ }^{46}$, pidiendo un voto en la confianza y voluntad de conseguir una Francia más justa, más igualitaria y más fraterna. Para ello reclama el voto de los verdes, a pesar de que su candidatura indicó que no daría consigna de voto para la segunda vuelta, sí manifestó que daría a conocer a sus votantes la posición del líder socialista en algunos temas que preocupan a los ecologistas; de los comunistas, cuyos dirigentes es-

44 Alrededor de un 20 por 100 de los votantes, según una encuesta publicada por Le Monde, 27 de abril de 1995.

45 El País, 26 de abril de 1995.

$46 \quad$ ABC. 24 de abril de 1995. 
timan que la situación no se presta a un acuerdo con los socialistas ${ }^{47}$, y de Voynet que ha dejado ver una preferencia clara por Jospin ${ }^{48}$.

En lo que a la reagrupación de la derecha se refiere, Balladur ha prometido su apoyo a Chirac, aunque exige que éste tenga en cuenta una parte de su programa ${ }^{49}$, pero en cualquier caso no logra alcanzar el 50 por 100 de los votos necesarios, por lo que se hace preciso el apoyo de De Villiers que ha anunciado su voto a Chirac, pero éste tampoco es suficiente, lo que determinará que sea Le Pen quien se convierta en árbitro de la situación electoral, de forma que, por primera vez en la $V$ República la extrema derecha y la ultraderecha nacionalista "estén en situación de imponer sus criterios o vender muy caro su posible apoyo electoral $1{ }^{50}$.

Se ha afirmado ${ }^{51}$ que el dato más importante de la primera vuelta es la fuerza de los extremos que determina el voto de protesta emitido por los franceses, pero en estos extremos se alza con fuerza Le Pen que tiene la llave para abrir la puerta al próximo Presidente de la República.

Esta situación ha determinado que Chirac haya tenido que modificar su discurso, pues es conocedor que sin el voto de Le Pen perdería la presidencia, pero también que su voto le podría hipotecar durante su mandato. En las listas de Chirac las posturas van desde la negativa de oferta al Frente Nacional -Alain Madelin - hasta el ofrecimiento de una reforma electoral a Le Pen, que con el sistema mayoritario pierde representación parlamentaria - Charles Pasqua-, pero en el fondo se mantiene latente la preocupación de que el abundante voto obrero recibido por Le Pen se vuelque en favor de Jospin.

A pesar de estas afirmaciones la segunda vuelta se presenta muy reñida, a pesar de que las encuestas ${ }^{52}$ ofrecen un 43 por 100 a Jospin frente al 57 por 100 que otorgan a Chirac, puesto que en los últimos momentos de la campaña el líder del Frente Nacional ha dejado libertad a sus electores (15 por 100) para que elijan entre ambos candidatos para la presidencia de la República.

\footnotetext{
${ }^{47}$ L'Humanité, 25 de abril de 1995.

48 El Mundo. 25 de abril de 1995.

49 Le Monde. 26 de abril de 1995.

5o Amorós, JM. ABC, 25 de abril de 1995.

51 Sahagún, F.: "La fuerza de los extremos". El Mundo, 25 de abril de 1995.

52 Publicada por Le Monde, el 24 de abril de 1994.
} 


\section{Resultados electorales}

Chaques Chirac será el quinto Presidente de la V República francesa. Un 51,9 por 100 de los franceses ha confiado en el candidato de centro-derecha, frente al 48,1 por 100 de votos obtenidos por Jospin en una jornada que ha alcanzado el 81,45 por 100 de participación, lo que supone una pequeña reducción de la abstención manifestada en la primera vuelta electoral y que, sin duda, ha favorecido a Chirac.

Con estos resultados se pone fin a catorce años de mandato socialista al frente de la República y se matizan las tensiones Presidencia-Gobierno que se han manifestado en dos períodos gubernamentales de presidencia socialista.

Aunque la ventaja entre ambos candidatos presidenciales es escasa, en torno a los 4 puntos, no es sin embargo el resultado más apretado de la V República, puesto que Giscard se impuso a Mitterrand en 1974 por 50,8 por 100 frente a un 49,2 por $100^{53}$, pero sí ha servido para poner de manifiesto que la diferencia es menor de lo que lo es la correlación de fuerzas entre la derecha y la izquierda francesa.

Según un sondeo realizado a la salida de las urnas y que se ha completado con una proyección de intención de voto ${ }^{54}$, los electores de la extrema derecha votaron por Chirac en un 39 por 100, por Jospin en un 17 por 100, y en blanco o nulo un 13 por 100 , siendo el 31 por 100 restante, votantes que se abstuvieron en la elección.

El candidato socialista Jospin no ha ganado, pero ha podido demostrar que, tras los sucesivos reveses del PSF, la orientación política francesa no se haya detenido en el centro-derecha, y este candidato, que partía de la nada, ha sido capaz, de un lado de reagrupar al socialismo en torno a un nuevo líder, $y$ de otro de aglutinar en torno a él las fuerzas de la izquierda.

Al tercer intento, al igual que le sucediera a Mitterrand, ha triunfado Chirac, a pesar del tremendo susto que para él supuso la primera vuelta, que dirigirá cómodamente, si es capaz de reagrupar a las fuerzas de centro y extrema derecha, al disponer su formación política de una amplia mayoría en el Parlamento.

El País. 8 de mayo de 1995. 